\title{
Nodding syndrome: recent insights into etiology, pathophysiology, and treatment
}

This article was published in the following Dove Press journal: Research and Reports in Tropical Medicine

\section{Amos Deogratius Mwaka' Jerome Roy Semakula ${ }^{2}$ Catherine $\mathrm{Abbo}^{3}$ Richard Idro ${ }^{4}$}

'Department of Medicine, School of Medicine, College of Health Sciences, Makerere University, Kampala, Uganda; ${ }^{2}$ Infectious Diseases Institute, Makerere University College of Health Sciences, Kampala, Uganda; ${ }^{3}$ Department of Psychiatry, College of Health Sciences, Makerere University, Kampala, Uganda; ${ }^{4}$ Department of Pediatrics and Child Health, College of Health Sciences, Makerere University, Kampala, Uganda
Correspondence: Amos Deogratius Mwaka

Department of Medicine, School of Medicine, College of Health Sciences, Makerere University, PO Box 7072,

Kampala, Uganda

Tel +256 772569996

Emailmwakaad@yahoo.com

\begin{abstract}
Nodding syndrome is an enigmatic neuropsychiatric and epileptiform disorder associated with psychomotor, mental, and physical growth retardation. The disorder affects otherwise previously normal children aged 3-18 years, with a slight preponderance for the male child. Nodding syndrome has been described in rural regions of some low-income countries in sub-Saharan Africa including northern Uganda, South Sudan, and a mountainous region of southern Tanzania. The cause of the disorder has hitherto eluded scientists. Neuroimaging studies show involvement of the nervous system with associated severe cortical atrophy in the affected children. The affected communities have generated a number of perceived causes including some conspiracy theories related to intentional poisoning of water sources and foods, and causes related to fumes and chemicals from ammunitions used during civil wars in the affected regions. From biomedical perspectives, the treatment of the affected children is geared towards symptoms control and rehabilitation. There is evidence that seizures and behavioral problems including wandering and episodes of aggressions are controllable with anticonvulsants, especially sodium valproate and antipsychotics. No treatments have proven effective in reversing the course of the disorder, and cure remains a distant goal. Community members have used indigenous medicines, cleansing rituals, and prayer interventions, but have not perceived any reasonable improvements. A randomized controlled clinical trial is ongoing in northern Uganda to test the efficacy and effectiveness of doxycycline in the treatment of nodding syndrome. The hypothesis underlying the doxycycline trial underscores the role of antigenic mimicry: that antibodies generated against an antigen of a microorganism that resides inside the black flytransmitted parasite, Onchocerca volvulus becomes directed against nervous tissue in the brain This paper reviews some of the recent advances in researches on the etiologies, pathophysiology, and treatment of nodding syndrome.
\end{abstract}

Keywords: nodding syndrome, epilepsy disorders, epilepsy, conspiracy theory, Onchocerca volvulus, Northern Uganda, South Sudan

\section{Introduction}

Nodding syndrome (NS) is a neurological medical disorder with epileptiform, psychiatric, and physical manifestations. The etiology and pathogenesis of the disorder are still poorly understood. The disorder was first documented in Tanzania in the early 1960 s. $^{1}$ In the 1980 s, a nodding syndrome-like disorder was reported in Liberia. ${ }^{2,3}$ While the cases reported in south Tanzania and Liberia were in endemic proportions, the disorder was described in epidemic proportions in the Republic of South Sudan in the early 1990s and in northern Uganda in about 1997 with a peak in $2007 .{ }^{4-9}$ 
Typically, NS affects children, with symptom onset between the ages of 3 and 18 years, and causes progressive cognitive dysfunction, neurological deterioration, stunted growth, and a characteristic nodding of the head. ${ }^{8,10,11}$ Despite extensive investigations in all three East African countries, very little is yet known about the cause of NS. The purpose of this narrative review is to present a concise note on the recent information and ongoing research hypotheses geared towards the understanding of this peculiar devastating illness.

\section{The causes of NS}

\section{Perceived causes}

Once an illness or any calamity of significant magnitude strikes a society, members of the given society often ask several key questions in an effort to understand the illness, possible causes, the name of the illness, how to avert the illness, the effective treatments, the impact of the illness on affected persons, and fears regarding the illness: the illness explanatory model. ${ }^{12,13}$ The illness explanatory model concept has been used by social and medical anthropologists to understand illnesses and how to respond to illnesses in ways that are acceptable and in line with the perceptions and belief systems of the affected society. The culture of any given society determines how they react to and cope with illnesses. ${ }^{14,15}$ Researchers have used the Kleinman illness explanatory model for understanding "new illnesses" and their impacts on society to investigate the perceptions and beliefs of the NS-affected communities. Studies have revealed a consistent association of the cause of NS with war, internal displacement, and food shortages among communities in Northern Uganda. ${ }^{16-18}$ Majority of community members in the affected regions believe that NS resulted from some conditions related to the civil war and the internally displaced person (IDP) camps where the population in northern Uganda and South Sudan were forced to live during the insurgency as a way of protecting them from rebel brutality. To many commentators however, the IDP camps offered no protection to the population but was a ripe venue for torment and sexual abuse. ${ }^{19}$ The populations in the IDP camps were equally uncomfortable and suspicious of the intentions of the governments for congesting them in the camps as the rebels would raid and loot them with impunity even when in the protected IDP camps. ${ }^{19-21}$ The illness now known as NS was first observed by the local communities in Kitgum in about 1997 and later in larger numbers in about 2007 when people were beginning to leave the camps. People have associated NS with poisons in food and cooking oil provided to them in the camps, fumes and chemicals from bombs and ammunitions, and drinking water that were deliberately poisoned. ${ }^{17}$

Second, a substantial proportion of the people in the affected communities have associated the etiology of NS with vengeful spirits of innocent people killed, especially by child-soldiers, during the prolonged civil conflicts. These dead bodies were in addition not buried according to the traditions of the people. These spirits therefore "feel" neglected and disrespected and yet they were killed innocently. It is thought that the spirits attack children aged 3-18 years for two reasons; the child-soldiers who were forced to kill them were about that age, and second, the children in this age group who are returning home from the camps played with the unburied remains/bones of the innocently slayed persons. ${ }^{17,22,23}$ The vengeful spirits therefore target the children in this age group. Related to the spirits, community members also think there is an element of retribution from their creator for evils that could have been committed by the forefathers and especially soldiers in the regimes before the current National Resistance Movement government. These retributions and curses from the creator come in the form of evil spirits and target children in villages where former soldiers who could have killed innocent people in other parts of Uganda lived. Families that participated in causing problems to other people in the villages are also viewed as targets for the evil spirits, hence their children contracting NS. ${ }^{17,23}$

Members of the affected communities, especially in northern Uganda, are aware of the association that health care professionals, researchers, and government propose between NS and the black fly that spreads river blindness. However, they do not believe that black flies are reasonably associated with NS because their communities have lived in the black flies-affected river banks from time immemorial and had never before experienced any illness similar to NS. They report the black flies cause skin and eye diseases but not NS. They say that the unconfirmed association of NS with black flies is an escape route and a worthless defense by the governments that could have ostensibly connived with the suppliers of poisoned foods and strange thick oils that could have been extracted from poisonous plants. ${ }^{23}$ Similarly in South Sudan, community members associated the onset of disease with internal displacement due to civil war. ${ }^{24,25}$

\section{Scientific hypotheses on etiology and pathophysiology}

In our review of studies that investigated the possible causes of NS, the main recurring hypotheses were infections of the brain with Onchocerca volvulus and NS being a complex 
autoimmune neuroinflammatory disease. The arguments for and against the plausibility of these hypotheses have been reviewed in detail elsewhere. ${ }^{26-31}$ We therefore will not belabor to discuss all the hypotheses advanced in the above citations but rather delve into the recent insights on pathophysiology of NS and ongoing researches pursuing the most plausible scientific hypothesis, the Onchocerciasis-related cross-reacting autoantibodies concept. A clinical trial is ongoing in northern Uganda, testing the hypothesis that NS is a neuroinflammatory autoimmune disorder related to antigenic mimicry between an $O$. volvulus protein or an aspect of $O$. volvulus endosymbiont, Wolbachia, which contributes to the survival and longevity of the $O$. volvulus nematode..$^{32,33}$ The central hypothesis in this trial is that if NS indeed results from and the condition worsens in direct proportion to the degree of neuroinflammation and autoimmune depositions into the central nervous system, then reducing the lifespan of the adult $O$. volvulus by killing the Wolbachia that supports its survival and reproduction would significantly lead to reduction in symptoms and perhaps reversal of the course of NS in affected children. Wolbachia is highly susceptible to tetracyclines. ${ }^{32}$ The current trial is using doxycycline to eliminate the Wolbachia and then follow-up the affected children to observe changes in symptoms and course of NS. ${ }^{33}$ The international scientific community are eagerly waiting for the outcome of this trial which serves both a diagnostic purpose by indirectly providing evidence about the pathophysiology of NS and curative treatment choice for NS. To date, patients with NS are treated symptomatically with anticonvulsants to which they show satisfactory improvement. ${ }^{34}$

\section{Treatments for NS \\ Perceived treatments}

The various perceptions regarding the causes of NS, including supernatural causes and deliberately sent evil spirits led some community members to seek care with traditional and spiritual healers. Recourse to traditional medicines was also prompted by the perceived treatment failures with medicines from health facilities and multiple barriers to health seeking at the health facilities. ${ }^{22}$ Affected members of the population sought care from multiple sources, hoping to eventually find cure. ${ }^{35}$ However, the use of the indigenous approaches also did not lead to durable relief from NS symptoms. In some cases, even children of the traditional healers and witchdoctors became affected with NS, leading to loss of hope in the indigenous practices including cleansing rituals. Community members who believe in the effectiveness of indigenous remedies expect the healers to provide the best of the remedies for themselves and their children. And so, if children of the healers also contract NS and relentlessly suffer from the same, then it is likely that the indigenous remedies could indeed not be of value in the management of NS. ${ }^{17,23}$

\section{Biomedical treatments}

There is yet no known cure for NS, mainly because of the inadequate understanding of the pathophysiology and the actual cause of NS. In Uganda, current treatment approaches for NS patients are based on a guideline developed as part of the Uganda Ministry of Health and World Health Organization (WHO) clinical response to the disease in $2012 .{ }^{36}$ These guidelines were developed by a multidisciplinary team including professionals from the Uganda Ministry of Health, WHO, Mulago and Butabika National Referral and Teaching hospitals, and academics from Makerere and Gulu Universities. The multidisciplinary team comprised nurses, epidemiologists and public health specialists, occupational therapists, nutritionists, pediatricians and a pediatric neurologist, physicians, psychiatrists, obstetricians, physiotherapists, and speech and language therapists. This guideline was adopted as the standard reference for use by NS-affected countries during the first international scientific conference on NS in Kampala, Uganda in August 2012. ${ }^{37}$ The recommended treatments are based on symptoms and rehabilitation measures to ensure optimal functioning by the affected children. ${ }^{36} \mathrm{~A}$ review of over 500 patients in more than ten NS treatment health facilities in northern Uganda revealed reasonable symptoms control with use of anticonvulsants, nutritional support, psychotropic medications, and physical therapies based on this treatment guideline. ${ }^{34}$ Since the observation that seizures in NS respond appropriately to anticonvulsants and because there are no known causes to target, the objective of treatment in NS has been to relieve symptoms, offer primary and secondary prevention for disability, and rehabilitation to improve function. ${ }^{34,36}$ The current anticonvulsant of choice in treatment of NS is sodium valproate (Valparin ${ }^{\circledR}$ 200mg, Torrent Pharmaceutical Ltd., Mehsana, India). Sodium valproate is also active against some behavioral difficulties like aggression and impulsive behavior. Sodium valproate is administered, starting with a dose of $10 \mathrm{mg}$ / $\mathrm{kg} / \mathrm{d}$ in two divided doses and then increasing the dose by 5 $\mathrm{mg} / \mathrm{kg} / \mathrm{d}$ until seizure control is achieved or the maximum dose of $40 \mathrm{mg} / \mathrm{kg} / \mathrm{d}$ in children is reached. Sodium valproate has been shown to improve overall burden of seizures in children with NS as well as improvements in background electroencephalogram (EEG). The second line antiepileptic agent is lamotrigine (Lamotrigine TEVA 100mg, TEVA UK 
Limited, Eastbourne, UK), given at a dose of $1-10 \mathrm{mg} / \mathrm{kg} / \mathrm{d}$ in 1-2 divided doses with a maximum of $200 \mathrm{mg}$ daily in children under 12 years and $400 \mathrm{mg}$ for children 12 years or older. ${ }^{34,36}$ In all NS-affected sub-Saharan African countries, WHO recommends use of anticonvulsants including carbamazepine (Tegretol ${ }^{\circledR} 200 \mathrm{mg}$, Novartis Farma SpA, Torre Annunziata, Italy) and sodium valproate. ${ }^{36-38}$ However, there are a couple of concerns with use of antiepileptic medicines in the treatment and control of symptoms for NS including that sodium valproate can lead to congenital malformations when used in pregnant women, yet many NS-affected females are entering the reproductive age. ${ }^{27}$ Second, the availability of the anticonvulsants in health facilities in Uganda, and most sub-Saharan African countries, is very limited. This treatment gap is likely to severely affect effectiveness of NS treatment and control programs in these countries. ${ }^{39-42}$ The ongoing clinical trial in Uganda, assessing the effectiveness of doxycycline in the treatment of NS, could elucidate the pathophysiology and revolutionize the treatment and control of NS in sub-Saharan African countries. ${ }^{33}$

\section{Conclusion}

Knowledge of the causative agents and/or pathogenesis of NS is necessary for development of specific drug therapy that could lead to cure of NS as well as inform strategies for prevention. Specific treatments for NS are urgently needed to reverse the miserable health states of the NSaffected children. Current treatments for NS include use of anticonvulsants, especially sodium valproate for seizure control, and behavioral modifications including agitation and aggression in affected children. While NS is still limited to a few regions of three countries in sub-Saharan Africa, the disorder could potentially cause an epidemic in other $O$. volvulus-endemic areas of the world, leading to an international catastrophe and untold miseries on a large scale. Further studies are therefore needed to unmask this peculiar illness and thereby forge a clear approach for the management and prevention of NS.

\section{Author contributions}

Amos Deogratius Mwaka and Jerome Roy Semakula conceived the study, reviewed the literature, and drafted the manuscript. Catherine Abbo and Richard Idro critically reviewed the manuscript for important intellectual content and refined the message. This manuscript is part of review of literature to update interpretation of data from the doxycycline clinical trial. All authors contributed toward data analysis, drafting and critically revising the paper and agree to be accountable for all aspects of the work. All authors read and approved the final manuscript and agreed on its submission.

\section{Disclosure}

The authors report no conflicts of interest in this work.

\section{References}

1. Aall-Jilek LM. Epilepsy in the Wapogoro tribe in Tanganyika. Acta Psychiatr Scand. 1965;41(1):57-86.

2. van der Waals FW, Goudsmit J, Gajdusek DC. See-ee: clinical characteristics of highly prevalent seizure disorders in the Gbawein and Wroughbarh clan region of Grand Bassa County, Liberia. Neuroepidemiology. 1983;2(1-2):35-44.

3. Goudsmit J, van der Waals FW, Gajdusek C. Epilepsy in the Gbawein and Wroughbarh clan of Grand Bassa county, Liberia: the endemic occurrence of'See-ee'in the native population. Neuroepidemiology. 1983;2(1-2):24-34.

4. Spencer PS, Palmer V, Jilek-Aall L. Nodding syndrome: origins and natural history of a longstanding epileptic disorder in sub-Saharan Africa. Afr Health Sci. 2013;13(2):176-182.

5. Spencer PS, Vandemaele K, Richer M, et al. Nodding syndrome in Mundri county, South Sudan: environmental, nutritional and infectious factors. Afr Health Sci. 2013;13(2):183-204.

6. Wasswa H. Ugandan authorities deal with a mysterious ailment that leaves people nodding continuously. BMJ. 2012;344:e349.

7. Dowell SF, Sejvar JJ, Riek L, et al. Nodding syndrome. Emerg Infect Dis. 2013;19(9):1374.

8. Sejvar JJ, Kakooza AM, Foltz JL, et al. Clinical, neurological, and electrophysiological features of nodding syndrome in Kitgum, Uganda: an observational case series. Lancet Neurol. 2013;12(2):166-174.

9. Landis JL, Palmer VS, Spencer PS. Nodding syndrome in Kitgum District, Uganda: association with conflict and internal displacement. BMJ Open. 2014;4(11):e006195.

10. Idro R, Opoka RO, Aanyu HT, et al. Nodding syndrome in Ugandan children - clinical features, brain imaging and complications: a case series. BMJ Open. 2013;3(5). pii: e002540.

11. Winkler AS, Friedrich K, Meindl M, et al. Clinical characteristics of people with head nodding in southern Tanzania. Trop Doct. 2010;40(3):173-175.

12. Kleinman A. Concepts and a model for the comparison of medical systems as cultural systems. Soc Sci Med (1982). 1978;12(2B):85-95.

13. Kleinman A. Patients and Healers in the Context of Culture. Berkeley, CA: University of California Press; 1980:104-118.

14. Helman CG. Culture, Health and Illness. 5th ed. London: Hodder Arnold, an imprint of Hodder Education, part of Hachette Livre UK; 2007:128-143.

15. Kleinman A, Eisenberg L, Good B. Culture, illness, and care: clinical lessons from anthropologic and cross-cultural research. Ann Intern Med. 1978;88(2):251-258.

16. Bukuluki P, Ddumba-Nyanzi I, David Kisuule J, Ovuga E, Lien L, Kaawa-Mafigiri D. Nodding syndrome in post conflict Northern Uganda: a human security perspective. Global Health Govern. 2012;6(1).

17. Mitchell KB, Kornfeld J, Adiama J, et al. Nodding syndrome in northern Uganda: overview and community perspectives. Epilepsy Behav. 2013;26(1):22-24.

18. Mutamba B, Abbo C, Muron J, Idro R, Mwaka A. Stereotypes on Nodding syndrome: responses of health workers in the affected region of northern Uganda. Afr Health Sci. 2013;13(4):986-991.

19. Branch A. Humanitarianism, violence, and the camp in Northern Uganda. Civil Wars. 2009;11(4):477-501.

20. Branch A. Gulu in War ... and Peace? The Town as Camp in Northern Uganda. Urban Stud. 2013;50(15):3152-3167.

21. Branch A. The violence of peace: ethnojustice in Northern Uganda. Dev Change. 2014;45(3):608-630. 
22. Mwaka AD, Okello ES, Abbo C, et al. Is the glass half full or half empty? A qualitative exploration on treatment practices and perceived barriers to biomedical care for patients with nodding syndrome in post-conflict northern Uganda. BMC Res Notes. 2015;8(1):386.

23. Buchmann K. 'You sit in fear': understanding perceptions of nodding syndrome in post-conflict northern Uganda. Global Health Action. 2014;7(1):25069.

24. Tumwine J, Vandemaele K, Chungong S, et al. Clinical and epidemiologic characteristics of nodding syndrome in Mundri County, Southern Sudan. Afr Health Sci. 2012;12(3):242-248.

25. Nyungura JL, Akim T, Lako A, Gordon A, Lejeng L, William G. Investigation into the nodding syndrome in Witto Payam, Western Equatoria state, 2010. South Sudan Med J. 2011;4(1):3-6.

26. Colebunders R, Hendy A, Nanyunja M, Wamala JF, van Oijen M. Nodding syndrome - a new hypothesis and new direction for research. Int J Infect Dis. 2014;27:74-77.

27. Idro R, Opar B, Wamala J, et al. Is nodding syndrome an Onchocerca volvulus-induced neuroinflammatory disorder? Uganda's story of research in understanding the disease. Int J Infect Dis. 2016;45:112-117.

28. Colebunders R, Suykerbuyk P, Jacob S, van Oijen M. Nodding syndrome, other forms of epilepsy, and the Nakalanga syndrome most likely directly or indirectly caused by Onchocerca volvulus. J Neurol Sci. 2017;372:439-440.

29. Johnson TP, Tyagi R, Lee PR, et al. Nodding syndrome may be an autoimmune reaction to the parasitic worm Onchocerca volvulus. Sci Transl Med. 2017;9(377). pii: eaaf6953.

30. Colebunders R, Njamnshi AK, Oijen M, et al. Onchocerciasis-associated epilepsy: from recent epidemiological and clinical findings to policy implications. Epilepsia Open. 2017;2(2):145-152.

31. Colebunders R, Titulaer MJ. Nodding syndrome: preventable and treatable. Sci Transl Med. 2017;9(377). pii: eaam8532.

32. Bouchery T, Lefoulon E, Karadjian G, Nieguitsila A, Martin C. The symbiotic role of Wolbachia in Onchocercidae and its impact on filariasis. Clin Microbiol Infect. 2013;19(2):131-140.
33. Anguzu R, Akun PR, Ogwang R, et al. Setting up a clinical trial for a novel disease: a case study of the Doxycycline for the Treatment of Nodding Syndrome Trial - challenges, enablers and lessons learned. Global Health Action. 2018;11(1):1431362.

34. Idro R, Namusoke H, Abbo C, et al. Patients with nodding syndrome in Uganda improve with symptomatic treatment: a cross-sectional study. BMJ Open. 2014;4(11):e006476.

35. Atim P, Ochola E, Ssendagire S, Rutebemberwa E. Health seeking behaviours among caretakers of children with nodding syndrome in Pader District-Northern Uganda: a mixed methods study. PLoS One. 2016;11(7):e0159549.

36. Idro R, Musubire KA, Byamah MB, et al. Proposed guidelines for the management of nodding syndrome. Afr Health Sci. 2013;13(2): 219-225.

37. World Health Organization. International Scientific Meeting on Nodding Syndrome; July 30 -August 1; 2012; Kampala, Uganda. Available from: http://www.who.int/neglected_diseases/diseases/Nodding_syndrom_Kampala_Report_2012.pdf. Accessed February 3, 2018.

38. World Health Organization. Humanitarian Health Action. World Health Organization Joins Other Partners to Support Nodding Disease investigations in Southern Sudan. Geneva: WHO; 2011. Available from: http://www.who.int/hac/crises/sdn/sitreps/10june2011/en/. Accessed February 3, 2018.

39. Korevaar DA, Visser BJ. Reviewing the evidence on nodding syndrome, a mysterious tropical disorder. Int J Infect Dis. 2013;17(3):e149-e152.

40. Chin J. Epilepsy treatment in sub-Saharan Africa: closing the gap. Afr Health Sci. 2012;12(2):186-192.

41. Mbuba CK, Ngugi AK, Newton CR, Carter JA. The epilepsy treatment gap in developing countries: a systematic review of the magnitude, causes, and intervention strategies. Epilepsia. 2008;49(9): 1491-1503.

42. Wilmshurst JM, Kakooza-Mwesige A, Newton CR. The challenges of managing children with epilepsy in Africa. Semin Pediatr Neurol. 2014;21(1):36-41.
Research and Reports in Tropical Medicine

\section{Publish your work in this journal}

Research and Reports in Tropical Medicine is an international, peerreviewed, open access journal publishing original research, case reports, editorials, reviews and commentaries on all areas of tropical medicine, including: Diseases and medicine in tropical regions; Entomology; Epidemiology; Health economics issues; Infectious disease; Laboratory

\section{Dovepress}

science and new technology in tropical medicine; Parasitology; Public health medicine/health care policy in tropical regions; and Microbiology. The manuscript management system is completely online and includes a very quick and fair peer-review system. Visit http://www.dovepress. com/testimonials.php to read real quotes from published authors. 